\title{
Network Management Architecture Approaches Designed for Mobile Ad hoc Networks
}

\author{
Said El brak \\ Laboratory LIST, ERIT \\ FST of Tangier, Morocco
}

\author{
Mohammed Bouhorma \\ Laboratory LIST, ERIT \\ FST of Tangier, Morocco
}

\author{
Anouar A. Boudhir \\ Laboratory LIST, ERIT \\ FST of Tangier, Morocco
}

\begin{abstract}
Mobile Ad hoc Networks (MANETs) play an important role in the pervasive computing to support a wide range of applications. MANETs are self-organizing networks formed spontaneously from a set of mobile devices without any pre-existing fixed infrastructure or administrative support. Network management plays a vital role in the well-being of any communications network. It becomes extremely important and crucial, in order to keep the whole network and application work properly. However, MANETs present several constraints; they are characterized by a dynamic environment and the scarcity of resources. These features make their management rather difficult and a challenging task. Furthermore, the traditional network management approaches become impractical for these networks. This paper provides an overview of the main existing management architecture approaches dedicated to mobile ad hoc networks, and raises their theoretical and practical limitations. And then discusses issues for building efficient management architecture adaptable to MANETs.
\end{abstract}

\section{General Terms}

Network Management, Network Management Architecture.

\section{Keywords}

MANETs, Management, SNMP, Policy-based Autonomic computing.

\section{INTRODUCTION}

Mobile ad hoc networks are a potential solution for providing communication infrastructure. This technology allows network nodes to communicate directly to each other (possibly in multihop) without the need for fixed infrastructure. Ad hoc networks are useful in many application environments like conferencing, personal area networks, emergency services, intelligent transportation systems and wireless sensor networks, etc. However, they present several constraints such as infrastructureless and dynamic environment, frequently changing and unpredictable topology, limited resources and nodes play several roles at the same time (server, client, and router), etc [1].

In this paper, we will focus on managing ad hoc networks. By classical definition, "network management is a process of controlling a complex data network so as to maximize its efficiency and productivity" [2]. This process involves two main management activities, monitoring and configuring. As shown in figure 1, the management process represents a function that takes as input both objectives, observer states, and eventually the old-settings configuration. It returns as output the proper configuration parameters applied to the managed system.
Through this loop control, we can see that management process is similar to the concept of an automatic control system.

Management applications in MANETs include network monitoring, configuration and control of network and node resources (like battery life and bandwidth). The research work addressing to monitoring resides in measuring performance metrics, collecting the measurement data and processing these measurements. The configuration of ad-hoc networks must be performed in a self-organized manner to ensure robustness and scalability. A basic expectation in configuring ad hoc networks lies on the auto-configuration of network devices including the allocation of their IP addresses [3]. Unlike traditional fixed networks, MANETs rely on wireless connections between mobile nodes, which mean high rate of disconnections between nodes, limited network and node resources. So, there is a great need for a new management architecture able to cope with MANETs constraints.

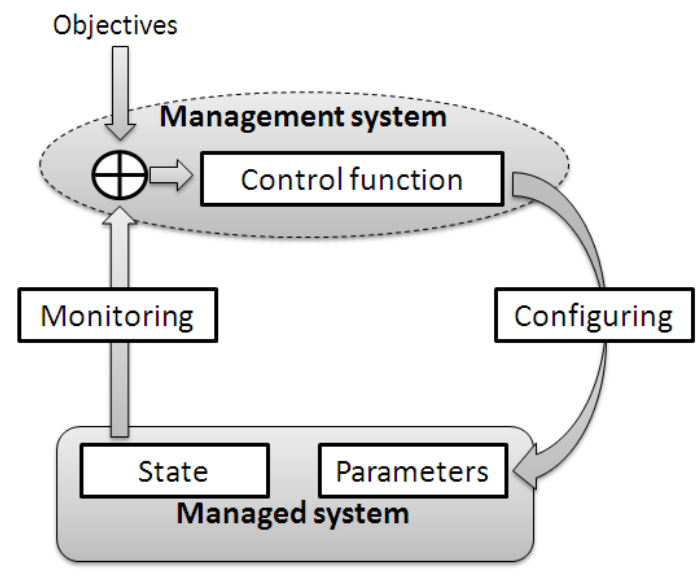

Fig 1: Management process

The rest of this paper is organized as follows: In section 2, we introduce the network management architecture (NMA), principals and proprieties of management architecture models presented in this section. Section 3 surveys the principal management architecture approaches for MANETs, it describes three main paradigms: SNMP-based, Policy-based and Selfmanagement approach. The next section 4 presents some discussions. Finally, we conclude the paper.

\section{NETWORK ARCHITECTURAL MODELS}

Designing network management system architecture is based on multidimensional modeling [2]. We consider three models that 
specify the conceptual aspect of information, organization and communication in the management architectures. Hence, we can define three models: the information model, the organizational model and the communication model. These models are not specific to ad-hoc networking but are recurrent in network management.

Information model defines a common formal framework for describing managed resources and the structuring of management information. It must offer a level of abstraction sufficient to provide homogeneous and scalable view of all resources, and this is irrespective of the nature, location and access methods. A resource is typically modeled by an object witch attributes indicate the state, and are accessible by management operations.

Organizational model defines the role (manager or agent) of each component of the management plan and specifies how each component interacts with the others. Network management systems can be classified according to their network organizational model.

- Centralized model: a single network manager acts as the manager station that collects information from all nodes and controls the entire network. The significant advantage of this model is to simplify the management task by considering a single point of control. However, this model has some problems like the incurring of high message overhead from data polling, and this limits its scalability.

- Distributed model: employs multiple manager stations. Each manager controls a subnetwork and may communicate directly with other manager stations. The usage of multiple managers reduces the overhead of management messages and improves the robustness of the management system.

- Hierarchical model: is a hybrid between the centralized and distributed models. Each local manager manages a subset of network nodes and is assigned with a given degree of responsibility.

Communication model specifies the protocol architecture to exchange management information between different entities. These include ensuring application-level exchanges between the manager and agent, in order to transmit operations and enable access and manipulation of data management.

Because of the nature of ad-hoc networks, these models need to be adapted. The role assignation and the relationships among components are highly dynamic in MANETs compared to regular fixed networks. The next section presents main trends for managing MANETs, and describes the underlying architectures able to cope with the constraints of such dynamic and resource scarce networks.

\section{MANETS NETWORK MANAGEMENT APPROACHES}

After the maturity of ad hoc routing protocols, which dominated initial research interest, the need for managing ad hoc networks come out as an important issue and is receiving important interest. Among the specific issues that a MANET management solution must address are: node positioning, network mobility, communication intermittence, node connectivity, network traffic, communication fairness, security and misbehavior of communication and routing [4]. We will present here three management approaches that have been proposed or redefined for the Ad-hoc networks.

\subsection{SNMP-Based:}

Current network management systems use a set of solutions. The Simple Network Management Protocol (SNMP) [5] is one of these solutions, which allows the exchange of management information between a network manager and the agents. SNMP is a widely common used protocol for fixed network management (monitoring and configuration). SNMP-Based approach focuses on developing a lightweight management protocol for MANETs that is compatible with SNMP protocol.

The Ad hoc Network Management Protocol (ANMP) [6] has been one of the first efforts and introduced an SNMP-based solution for MANETs. The compatibility with SNMP relies on using the same protocol data unit structure and the same management information base (MIB) structure. The major difference comes from the architectural model presented in figure 2, which combines clusters and hierarchical levels. ANMP comes within the scope of management by delegation. It clusters the management plane and organizes it in a three-level hierarchy composed of: individual managed nodes interacting as agents at the lowest level, clusterheads interacting as local managers at the middle level and a central network manager at the top level. The aim of this hierarchical model is to reduce the network traffic overhead by collating management data at the intermediate levels.

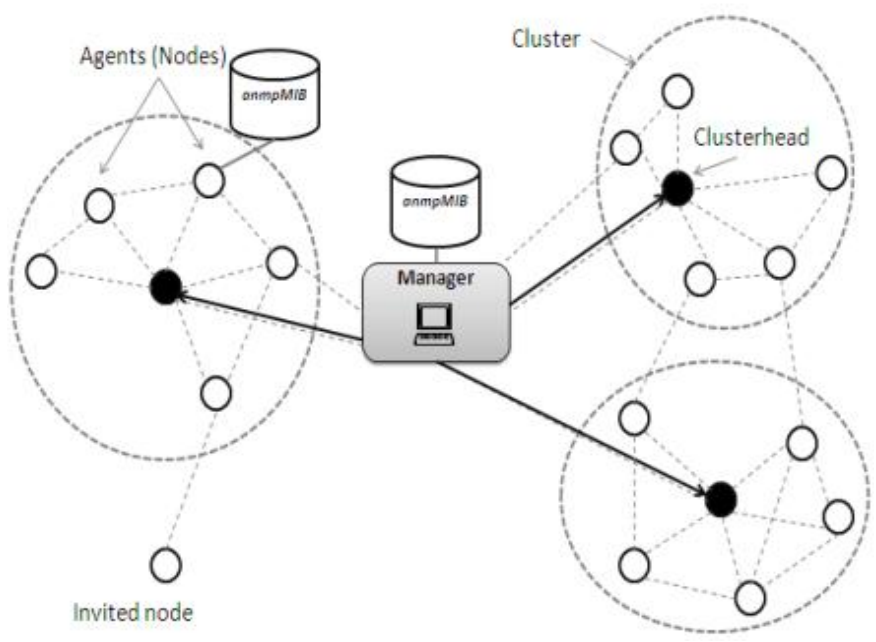

Fig 2: ANMP Architecture

To simplify the task of management, ANMP proposes two clustering algorithms: graph-based algorithm and geographicalbased algorithm. The first approach models the ad hoc network graph-based and form clusters based on the graph topology. The second technique uses GPS information to divide the network into clusters. ANMP makes a distinction between clustering at the application layer for management purposes and clustering at the routing layer.

anmpMIB is a management information base defined by ANMP specific to MANETs as an additional group to the standardized MIB-II of SNMP. The group called Level-based Access Control 
Model (LACM) includes security information data in order to secure management operations and management information access.

An alternative SNMP-based solution is proposed in [7] by GUERRILLA Management Architecture (GMA). Uses the cluster based management mechanism and mobile agents in order to implement an autonomic management environment. Nodes are clustered into groups with at least one nomadic manager in each group. The nomadic managers collaborate autonomously to manage the entire network.

However, a problem with utilizing SNMP-based management architecture approaches in MANETs is the cost of maintaining a hierarchy (cluster construction and cluster head election process) or to disseminate requests and collect replies in the face of node mobility. Thus, introduce an additional overhead that will increase energy consumption and decrease the available bandwidth.

\subsection{Policy-Based}

Traditional approaches to network management focus on individual devices and often rely upon proven technologies, such as the SNMP protocol. However, it can be quite a time consuming resources if the number of managed nodes is great. Policy-based network management (PBNM) [8] simplifies the complex management tasks of large scale systems, since policies monitor the network and automatically enforce appropriate actions in the system. However, policies specify the conditions that should be satisfied before executing management operations for any desired goals. Generally, policies are roles in the general form: ON <event> IF <condition> DO <action>.

The policy-based management system has the following components (Figure 3): a policy enforcement point (PEP), a policy decision point (PDP), a policy repository (PR), and a policy management tool. The task of the PDP is to retrieve and interpret the policy information, and pass it to the PEP. The policy repository is the place where all policies are stored and from which they are taken by PDPs.

The Common Open Policy Service (COPS) [9] protocol was specified to standardize the distribution policy through the PDPPEP communications. COPS have found little acceptance and their relatively heavyweight nature may limit their applicability to MANETs.

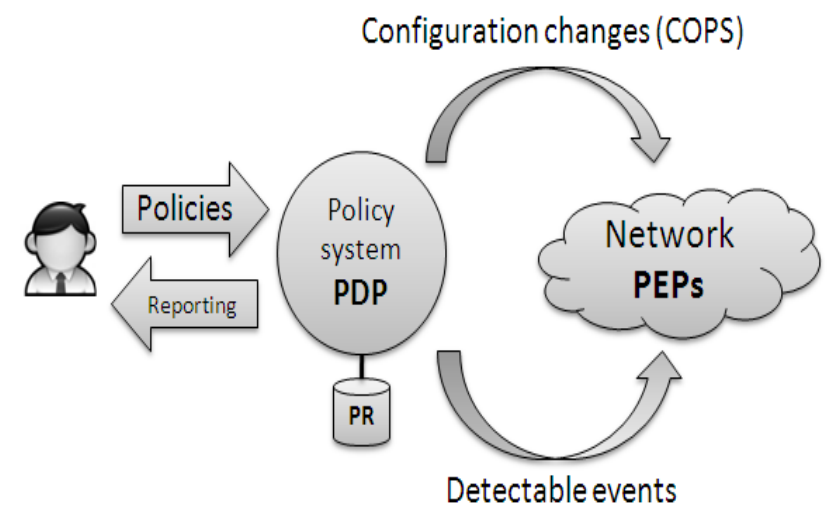

Fig 2: PBNM System Architecture
DRAMA [10] is a PBNM system for MANETs, using intelligent agents. Policy agents are deployed and manage the network through a two tier hierarchical architecture (figure 4). Global policy agent (GPA) manages multiple Domain Policy Agents (DPAs). DPA can manage multiple DPAs or Local Policy Agents (LPAs). LPA manages a node. LPAs perform local policy-controlled configuration, monitoring, filtering, aggregation, and reporting. Thus reducing management bandwidth overhead. DRAMA uses Yelp Announcement Protocol (YAP) for efficiently reporting management information. Policies are distributed using a combination of DRCP/DCDP (Dynamic Configuration Distribution Protocol/ Dynamic and Rapid Configuration Protocol).

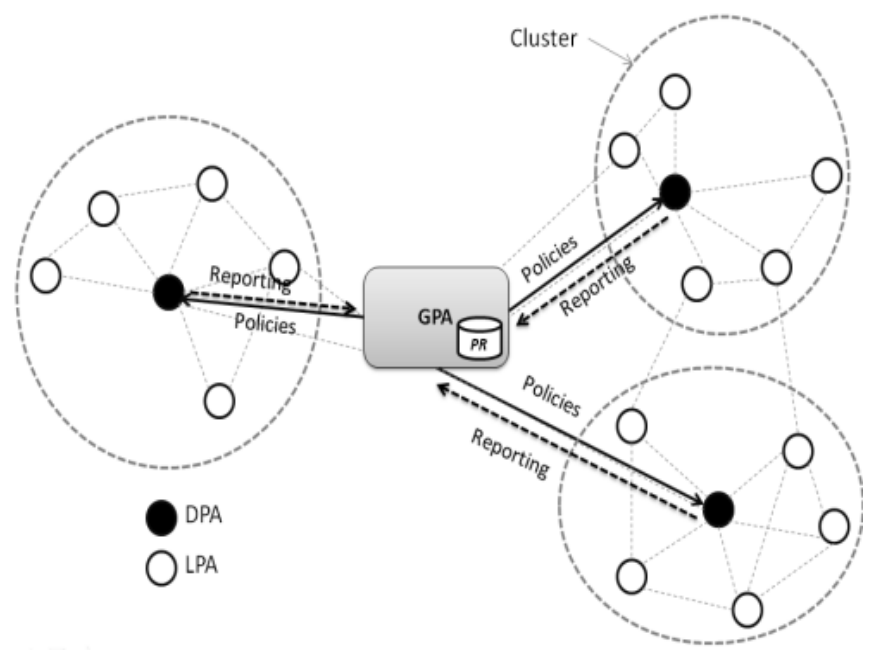

Fig 3: DRAMA Architecture

DRAMA management system was prototyped and demonstrated in a realistic environment to illustrate several use cases including CPU utilization reporting, server reallocation upon failure and reconfiguration of bandwidth allocation. The conceptual architecture of DRAMA is relatively simple, but allows an exhaustive set of experiments.

\subsection{Self-managing based}

The self-managing approach allows systems to manage themselves by given high-level objectives from administrators. This approach is based on autonomic computing paradigm that has been proposed by IBM in 2001[11]. IBM has defined four general properties a system should have to constitute selfmanagement:

- self-configuring: the ability of the system to configure and reconfigure automatically itself under varying conditions and changes in its environment,

- $\quad$ self-optimizing: the ability of the system to optimize efficiently its use of the resources by measuring and tuning them,

- self-healing: the ability of the system to detect instantly improper events and react to system disruptions,

- self-protecting: the ability of the system to detect, identify and protect against attacks. 
Autonomic network architecture consists of a collection of autonomic elements. As depicted in figure 5, each autonomic element is typically consisting of an autonomic manager and one or managed element. Managed element could be a resource such as storage, CPU, etc. Autonomic manager is composed of:

- Monitor: which is responsible for knowledge gathering,

- Knowledge base: which consists of a repository where policies and monitored information are stored,

- "Analyze and Plan" component: which analyzes knowledge and constructs plans of actions,

- Executor: which reconfigures the system regarding to the output of analyze and plan processes.

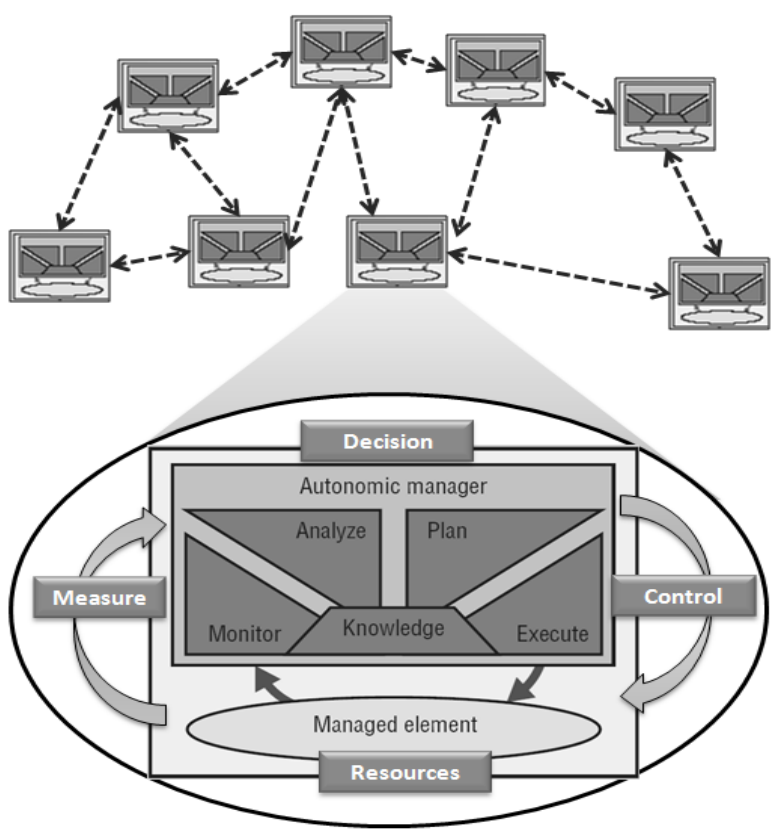

Fig 4: Autonomic Control Loop

Autonomic computing seems very useful in the case of MANETs, because these networks are self-creating and need to be self-managing. The application of the autonomic paradigms on MANETs is recent. In [12] Autonomous Decentralized Management Architecture (ADMA) has been proposed. This solution improves the self-configuring capabilities in MANETS by totally distributing policy-based management system. ADMA gives the system the ability to configure and reconfigure itself under varying conditions and changes in its environment without a human intervention.

The originality of ADAM is that each node contains its local component defined by PBNM Architecture: LPDP, PEP, PR and a monitor (figure 6). Hence, all nodes are able to take the appropriate decision based on predefined high level policies and collected monitoring information. Therefore, the role of the network operator is limited to define high level policies and introduce them to at least one node of the network. These policies will be automatically distributed throughout the network and to new arriving nodes. ADMA distinguished four classes of predefined policies: configuration, reconfiguration, monitoring and meta-policies. To distribute policies over the network or node entities (LPDP, PEP and Monitor), ADMA propose a Distributed Policy Management Protocol (DPMP) [13] which supports the internal communication between LPDP, PEP and the monitor components, as well as inter-LPDP communication.

DPMP has been implemented and evaluated under ns-2, and the performance of the designed solution was tested in the case of a real-time application (Voice over IP). The simulation results show an improvement of the VoIP delay and loss rate.

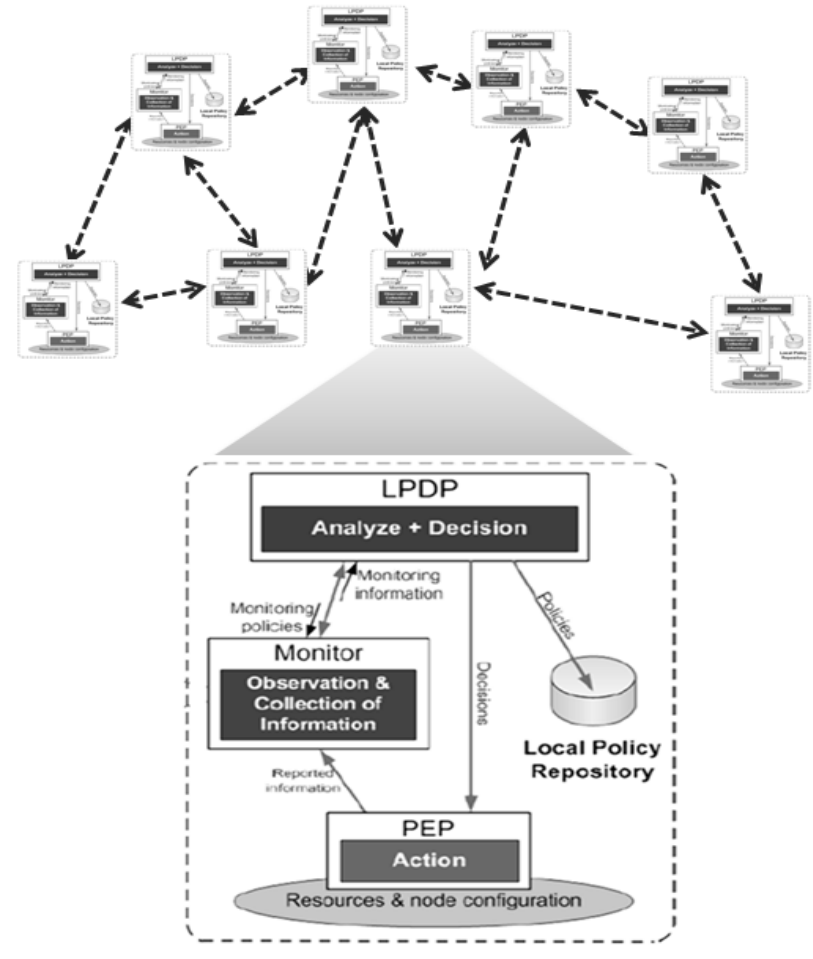

Fig 5: ADMA node architecture

\section{DISCUSSION}

Given the above overview, MANETs management architecture approaches have different limits. Hierarchical management model based on SNMP can perform complex management task and more accurate management decisions. Although, it has several disadvantages like: high message overhead, single point failure and in partitioned network some nodes left without any management functionality. Because of the self-organized characteristic of MANETs, the management task has to be distributed. Policy-based network management offers relatively this feature, by executing and applying policies previously defined by network manager. Otherwise, the complexity of realization and control makes its realization rather difficult. Autonomic network trend proposes the self-management solution. This approach leads to a NMA able to take into consideration the autonomous nature of MANETs. A drawback of the self-management approach is that it may be too computationally for resource constrained nodes and it needs sensor nodes to be dedicated for specific management roles.

In general, MANETs network management architecture must has several criteria like: Lightweight, Robustness and Fault 
Tolerance, Adaptability and Responsiveness, Minimal node resource usage and Control of Functionality and Scalability. Up to now, there is no NMA that supports all these features. Thus, the specific requirements that a MANET management architecture solution must address are depending on the nature of network application domain. Specific characteristics of the application need to be translated into a number of significant factors that must be considered in coming up with approaches to the management of these networks. For example, the network lifetime should be an important factor in managing wireless sensors network application. Topology and mobility are the most factors in managing vehicular ad hoc networks. Therefore, we can say that MANET network management architectures are application dependent.

One of the crucial issues that the NMA designers deal with is the management message overhead. Therefore, they need to exploit the routing plan to provide information and communication for the management system. Routing protocol (e.g. OLSR, AODV) can be utilized to report monitoring management information or configuration parameter, these can reduce management message overhead and enhance the performance of MANET.

A NMA designed for MANETs should promote a set of management services like resource productivity and improve the performance of the network. Otherwise, it should not be hyperconsumer of network and nodes resources. Hence new metrics need to be defined to evaluate a given NMA solution. For instance we can specify the NMACost (Network Management Architecture Cost) which can be defined as the ratio of the services provided by the NMA and consumed resources.

\section{CONCLUSION}

In conclusion, we can say that none of the current approach provides a fully integrated view of all MANETs management design factors. Furthermore, most of these systems incorporate management functions within application protocols. The development of general purpose network management architecture is a challenging task. Accordingly, this paper discusses management issues in MANETs and proposes some techniques like to integrate the management architecture with routing plan and define new metric to evaluate the network management architecture. This paper reviewed the main network management architecture approaches proposed for mobile ad hoc networks. It is beneficial for researcher and beginners of this domain.

\section{REFERENCES}

[1] C.K. Toh, Ad-Hoc Mobile Wireless Networks. Number ISBN 0-13-007817-4. Pearson Education, Prentice Hall, New Jersey, USA, 2002.
[2] H.G. Hegering, S. Abeck, and B. Neumair. Integrated Management of Networked Systems: Concepts, Architectures, and Their Operational Application. Morgan Kaufmann Publisher, 1999.

[3] R. Badonnel, Management of Ad-Hoc Networks and Services. Thésis, LORIA - INRIA Lorraine, 2007.

[4] C. Shen, C. Srisathapornphat, and C. Jaikaeo, An Adaptive Management Architecture for Ad hoc Networks, IEEE Communications Magazine, Vol. 41, No. 2, pp. 108-115, Feb. 2003.

[5] J. Case, M. Fedor, M. Schoffstall, and J. Davin, RFC 1157 - A Simple Network Management Protocol (SNMP), Network Working Group, Request for Comments 1157, 1990.

[6] W.Chen, N.Jain, and S.Singh, "ANMP: Ad Hoc Network Management Protocol", IEEE Journal on Selected Areas in Communications, Vol. 17, No. 8, August 1999

[7] C. Shen, C. Jaikaeo, C. Srisathapornphat, and Z. Huang, The GUERRILLA Management Architecture for Ad-hoc Networks. (MILCOM'02), Anaheim, CA, USA, October 2002.

[8] D.C. Verma and D. Verma, Policy-Based Networking: Architecture and Algorithms. New Riders Publishers, November 2000.

[9] D. Durham, J. Boyle, R. Cohen, S. Herzog, R. Rajan, and A. Sastry. The COPS (Common Open Policy Service) Protocol.

http ://www.ietf.org/rfc/rfc2748.txt, January 2000. IETF Request for Comments 2748.

[10] Ritu Chadha, Cho-Yu Jason Chiang." DRAMA: Distributed Policy Management for MANETs" pp.235-237, 2008

[11] N. Agoulmine, S. Balasubramaniam, D. Botvich, J. Strassner, E. Lehtihet, and W. Donnelly. Challenges for autonomic network management. In 1st IEEE International Workshop on Modelling Autonomic Communications Environments (MACE) Proceedings. TeX Users Group, Dublin, Ireland, October 2006.

[12] M.Ayari , Z.Movahedi, F.Kamoun ” ADMA: Autonomous Decentralized Management Architecture for MANETs - A Simple Self-Configuring Case" IWCMC'09, June 21-24, Leipzig, Germany, 2009.

[13] M. Ayari, F. Kamoun, and G. Pujolle. Distributed policy management protocol for self-configuring mobile ad-hoc networks. Advances in Ad Hoc Networking, 265: pp. 7384, August 2008. 